\title{
Breathers and thermal relaxation in Fermi-Pasta-Ulam arrays
}

\author{
Ramon Reigada \\ Departament de Química Física, Universitat de Barcelona, Avda. Diagonal 647, 08028 Barcelona, Spain
}

Antonio Sarmiento

Instituto de Matemáticas, Universidad Nacional Autónoma de México, Ave. Universidad s/n, 62200 Chamilpa, Morelos, México

Katja Lindenberg

Department of Chemistry and Biochemistry and Institute for Nonlinear Science, University of California San Diego, 9500 Gilman Drive, La Jolla, California 92093-0340

(Received 14 October 2002; accepted 21 November 2002; published 22 May 2003)

\begin{abstract}
Breather stability and longevity in thermally relaxing nonlinear arrays depend sensitively on their interactions with other excitations. We review numerical results for the relaxation of breathers in Fermi-Pasta-Ulam arrays, with a specific focus on the different relaxation channels and their dependence on the interparticle interactions, dimensionality, initial condition, and system parameters. () 2003 American Institute of Physics. [DOI: 10.1063/1.1537090]
\end{abstract}

\begin{abstract}
Breathers are highly localized oscillatory excitations in discrete nonlinear lattices that have been invoked as a possible way to store and transport vibrational energy in a large variety of physical and biophysical contexts. A particular scenario where the robustness and longevity of breathers has been a matter of considerable debate involves nonlinear arrays subject to thermal relaxation via the connection of surface sites to a cold environment. The important questions are these: Can breathers (created spontaneously or by design) survive for a long time in such a relaxing environment? If they can survive, can they move? We detail answers to these questions, one of which is rather unequivocal: Breathers that move do not live very long. So is another: Breathers are quite robust when they do not move. The more complicated question then deals with the conditions that allow breathers to remain stationary and undisturbed for a long time in a relaxing environment. We detail some conditions that lead to this outcome, and others that definitely do not.
\end{abstract}

\section{INTRODUCTION}

The localization of vibrational energy in discrete nonlinear arrays has attracted a huge amount of interest in the past several decades as a possible mechanism for the efficient storage and transport of energy (for recent reviews see Refs. 1 and 2, and references therein). More recently, the localization and transport of vibrational energy has been invoked in a number of specific physical settings including DNA, ${ }^{3}$ hydrocarbon structures, ${ }^{4}$ the creation of vibrational intrinsic localized modes in anharmonic crystals, ${ }^{5}$ photonic crystal waveguides, ${ }^{6}$ and targeted energy transfer between donors and acceptors in biomolecules. ${ }^{7}$

Discrete nonlinear arrays in thermal equilibrium can support a variety of stationary excitations; away from equilibrium stationarity may turn into finite longevity, and additional excitations may arise. The possible excitations include phonons associated with linear portions of the potential, solitons $^{8,9}$ (long-wavelength excitations that persist from the continuum limit upon discretization), periodic breathers ${ }^{1,2,9-13}$ (spatially localized time periodic excitations that persist from the anticontinuous limit upon coupling), and so-called chaotic breathers ${ }^{11}$ (localized excitations that evolve chaotically). Nonlinear excitations have been observed to arise (spontaneously or by design) and survive for a long time in numerical experiments, and they clearly play an important role in determining the global macroscopic properties of nonlinear extended systems.

Of particular interest to us is the dynamics of breathers in a chain relaxing to zero temperature. We invoke the term "breather" rather loosely to denote an oscillatory excitation confined to a very small number of adjacent lattice sites. Since our interest lies in breathers as possible storers and carriers of energy, we have concentrated on issues of longevity, and on lattices where breathers can move most easily even while the entire system is relaxing to zero temperature. Breathers are known to move more easily in nonlinear lattices with no on-site interactions, and so we have focused on lattices with nonlinear interactions. In Ref. 14 we studied the relaxation to zero temperature of one-dimensional chains initially thermalized at a finite temperature. Among other issues, we investigated the effects of different types of interactions. In Ref. 15 we extended these studies to two dimensions, and found that while many relaxation properties are insensitive to dimensionality, in some cases there are important differences. In both, we were principally interested in the spontaneous appearance and evolution of breathers during the overall decay process. In Ref. 16 we focused on the very long time relaxation behavior of breathers (injected or created spontaneously) and found profound differences in breather longevity in systems with and without a linear interaction component. Herein we organize these results into a rather complete picture of the breather relaxation. Even more narrowly, we focus on the nonequilibrium dynamics and decay of breathers in a typical relaxation experiment where the surface of the system is connected to a cold (usually zero 
temperature) external thermal reservoir. We mostly (but not exclusively) study one-dimensional arrays, for which the surface simply consists of the two end sites of a finite chain. We review and organize our previous work, and also include new results that complement our previous ones.

We anticipate, and later detail, the following broad-brush description of the relaxation of a breather whose energy is well above that of phonon modes that may also be present in the nonlinear array. When the array boundaries are connected to a zero-temperature heat bath, the breather will of course eventually decay since the system must reach equilibrium at $T=0$. In other words, there is necessarily leakage of energy out of the breather, although this process may in some cases be extremely slow. A determinant limiter of breather longevity is the extreme sensitivity to collisions with long wavelength phonons and with other localized excitations. Such collisions invariably contribute to the rapid degradation or breakup of breathers into lower energy excitations. Furthermore, collisions with other excitations tend to set breathers in motion, and motion in itself also contributes to energy leakage. While breathers tend to decay rapidly in the presence of long wavelength phonons and of other nonlinear excitations, and are in this sense fragile, isolated breathers tend to remain stationary and to decay extremely slowly and essentially exponentially over long time regimes, indicating a single slow rate-limiting dominant contribution to the intrinsic relaxation process. However, the particular values of decay rates are strongly sensitive to particular conditions and parameter values. These statements will be made more quantitative in the following.

The organization of this paper is as follows. The model is presented in Sec. II, and a summary of the relaxation of phonon modes in harmonic lattices ${ }^{17}$ is presented in Sec III. In Sec. IV we discuss the relaxation behavior of a purely anharmonic lattice (no harmonic interactions), that is, a relaxation scenario that involves only nonlinear excitations and no phonons. Section V deals with breather relaxation in arrays with both linear and nonlinear interactions, that is, lattices that support phonons as well as nonlinear excitations. Finally, we present a summation of our findings in Sec. VI.

\section{THE MODEL}

Our model system in one dimension is described by the Fermi-Pasta-Ulam (FPU) $\beta$-Hamiltonian

$$
H=\sum_{i=1}^{N} \frac{\dot{x}_{i}^{2}}{2}+\sum_{i=1}^{N} V\left(x_{i}-x_{i-1}\right),
$$

where $x_{i}$ is the displacement of particle $i$ from its equilibrium position, $N$ is the number of sites, $V(z)$ is the FPU potential

$$
V(z)=\frac{k}{2} z^{2}+\frac{k^{\prime}}{4} z^{4}
$$

and $k$ and $k^{\prime}$ are the harmonic and anharmonic force constants, respectively. The generalization to higher dimensions is obvious. The relative values of the two constants can be shifted by rescaling space and time. In particular, by introducing new variables $y_{i}=\alpha x_{i}$ and $\tau=t / \alpha$, where $\alpha$ is a scal- ing constant, one finds that the scaled Hamiltonian $\alpha^{4} H$ in the new variables is again of the form (1) but with coupling constants $\alpha k$ and $k^{\prime}$. The results are therefore related through appropriate scaling for any choice of coupling constants provided neither is zero. To cover all possible combinations of coupling constants it is thus sufficient to consider only three distinct cases: $k^{\prime}=0$ (harmonic), $k=0$ (purely anharmonic), and $k=k^{\prime}$ (mixed). Throughout we assume free-end boundary conditions $\left(x_{0}=x_{1}, x_{N+1}=x_{N}\right)$, and note that although boundary conditions do not strongly affect equilibrium properties, they do affect relaxation dynamics.

The equations of motion associated with the Hamiltonian (1) are

$$
\ddot{x}_{i}=-\frac{\partial}{\partial x_{i}}\left[V\left(x_{i}-x_{i-1}\right)+V\left(x_{i+1}-x_{i}\right)\right] .
$$

In our subsequent discussion we consider a variety of initial conditions, and observe the relaxation of the array to zero temperature when the boundary sites are connected to a zerotemperature environment by adding dissipation terms $-\gamma \dot{x}_{i}$ to the equations of motion of these sites. In one dimension the boundary sites are $i=1$ and $i=N$. The equations of motion are integrated using a fourth-order Runge-Kutta method with time interval $\Delta t=5 \times 10^{-4}$. Further reduction leads to no significant improvement. Stability of the integration was checked for isolated arrays: the energy remains constant to 10 or more significant figures for all the cases and over all time ranges reported herein.

\section{LINEAR MODES}

In the absence of anharmonic interactions the excitations of the system are phonons whose behavior is well known. It is useful to briefly review this behavior because phonons may be present in the nonlinear system, and their presence strongly affects the relaxation behavior of nonlinear excitations.

There are two informative measures to characterize the relaxation behavior of an array initially thermalized ${ }^{18}$ at temperature $T$ and then allowed to relax through the array boundaries into a zero temperature heat bath. ${ }^{14,15}$ One is the total array energy as a function of time, and the other is the time-dependent spectrum. The total energy $\varepsilon(t)$ is defined as the sum over symmetrized site energies, e.g., in one dimension

$$
\begin{aligned}
& \varepsilon(t)=\sum_{i=1}^{N} E_{i}(t), \\
& E_{i}(t)=\frac{p_{i}^{2}}{2 m}+\frac{1}{2} V\left(x_{i+1}-x_{i}\right)+\frac{1}{2} V\left(x_{i}-x_{i-1}\right) .
\end{aligned}
$$

The time-dependent spectrum is the Fourier transform of the time-dependent correlation function,

$$
S(\omega, t)=2 \int_{0}^{\tau_{\max }} C(\tau, t) \cos (\omega \tau) d \tau,
$$

where $\tau_{\max } \equiv 2 \pi / \omega_{\min }$ and $\omega_{\min }$ is chosen for a desired frequency resolution. The choice $\omega_{\min }=0.0982$ (corresponding 
to $\tau_{\max }=64$ ), turns out to be numerically convenient. In one dimension the time-dependent correlation function is

$C(\tau, t)=\frac{1}{(N-1)} \sum_{i=2}^{N} \frac{1}{\Delta t} \int_{0}^{\Delta t}\left\langle\Delta_{i}\left(t-\tau^{\prime}\right) \Delta_{i}\left(t-\tau^{\prime}-\tau\right)\right\rangle d \tau^{\prime}$,

where $\Delta_{i}=x_{i}-x_{i-1}$ is the relative displacement and $\Delta t \equiv t_{0}$ $-\tau_{\max }$. The correlation function is thus an average over the interval $\left[t-t_{0}, t\right]$, and $t_{0}$ is a time interval chosen to be short enough for the correlation function not to change appreciably but long enough for statistical purposes. In our work we take $t_{0}=100$. The generalization of these definitions to higher dimensions is straightforward.

If the chain is sufficiently long and the damping constant $\gamma$ at the ends sufficiently small, then the phonon dynamics in the array are not greatly disturbed by the damping, and the relaxation process is perturbative. The two principal characteristics of phonon relaxation are then that (1) phonons of each frequency relax independently of other phonons, and (2) the relaxation times are wave vector dependent. The relaxation time for phonons of wave vector $q$ in the small damping limit has been calculated by Piazza et al. ${ }^{17}$ for different boundary conditions. For free-end boundaries they obtain the decay times in one dimension $\tau(q)=\tau_{0} / \cos ^{2}(q / 2)$, where $q=n \pi / N, n=0,1, \ldots, N-1$ are the allowed wave vectors, and $\tau_{0}=N / 2 \gamma$. Long wavelength phonons thus decay more rapidly $[\tau(q) \sim \mathcal{O}(N / \gamma)]$ than do band edge phonons $\left[\tau(q) \sim \mathcal{O}\left(N^{3} / \gamma\right)\right]$. The associated normalized chain energy $E(t)$ as a function of time can be evaluated exactly in this limit:

$$
\begin{aligned}
E(t) \equiv \frac{\varepsilon(t)}{\varepsilon(0)} & =\frac{1}{\pi} \int_{0}^{\pi} e^{-2 t / \tau(q)} d q \\
& =e^{-t / \tau_{0} I_{0}\left(t / \tau_{0}\right)} \\
& = \begin{cases}e^{-t / \tau_{0}} & \text { for } t \ll \tau_{0} \\
\left(\frac{2 \pi t}{\tau_{0}}\right)^{-1 / 2} & \text { for } t \gg \tau_{0} .\end{cases}
\end{aligned}
$$

Here $I_{0}$ is the modified zero-order Bessel function. The short time exponential behavior reflects the earliest decay of the long wavelength phonons. The power law behavior at long times arises from the cascade of relaxation times that contribute to the process. In a finite chain at very long times the decay will revert to exponential when only the shortest wavelength phonons remain, with a characteristic decay time of $\mathcal{O}\left(N^{3} / \gamma\right)$. Similar arguments are immediately applicable in higher dimensions. Note that the energy decay in the harmonic arrays is independent of the initial temperature. The decay curve for a one-dimensional harmonic chain with $\gamma$ $=0.1$ is shown in Fig. 1. A typical time-dependent landscape in which energy magnitudes are represented by varying gray scales has been presented in our earlier work. ${ }^{14,15}$

It should be recognized that the above-reported results are restricted to weak damping. If the damping coefficient becomes large $(\gamma \gg \sqrt{4 k}=\sqrt{2})$, the theory of Piazza et al. no longer applies. The boundaries act more like hard walls and the phonon decay slows down with increasing $\gamma$. As an ex-

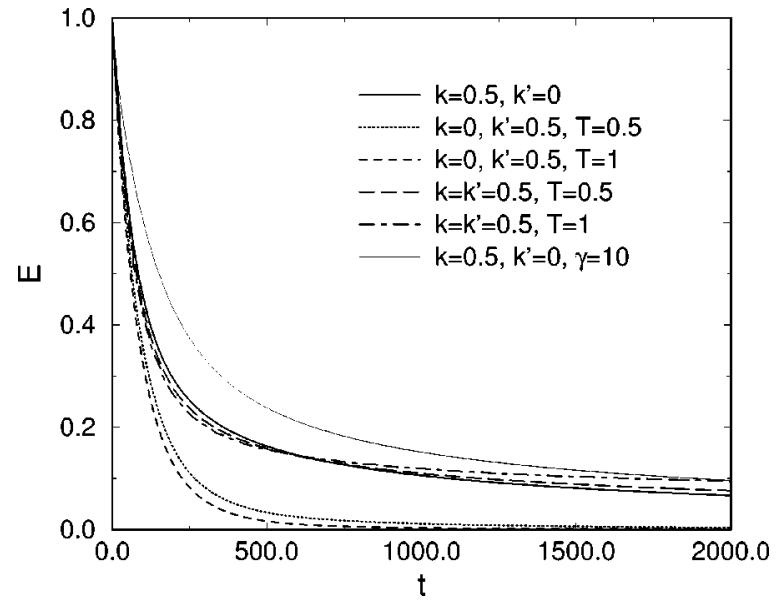

FIG. 1. Normalized energy vs time for various one-dimensional relaxing arrays with $N=50$. Initially each array is in thermal equilibrium at the temperature indicated. The normalized energy of the harmonic array is independent of temperature. In all cases except for the thin solid line, $\gamma$ $=0.1$. The thin solid curve is for the harmonic chain with $\gamma=10$.

ample we have included the one-dimensional (1D) curve for $\gamma=10$ in Fig. 1, which is clearly qualitatively similar to the $\gamma=0.1$ curve. Although not shown, we note that the decay curves of the normalized energies for the damping parameters $\gamma=0.01$ and $\gamma=100$ are also quite similar to one another. Note that the relaxation slows down with increasing damping. The concept of "optimal damping" that this behavior implies is intriguing.

The spectral progression of the phonon-by-phonon decay in the weak damping case is interesting because it provides a forum to differentiate linear from nonlinear systems in this limit. The first panel of Fig. 2 shows the time progression of the spectrum of an initially thermalized harmonic chain as it relaxes. The $t=0$ curve is the equilibrium spectrum of the

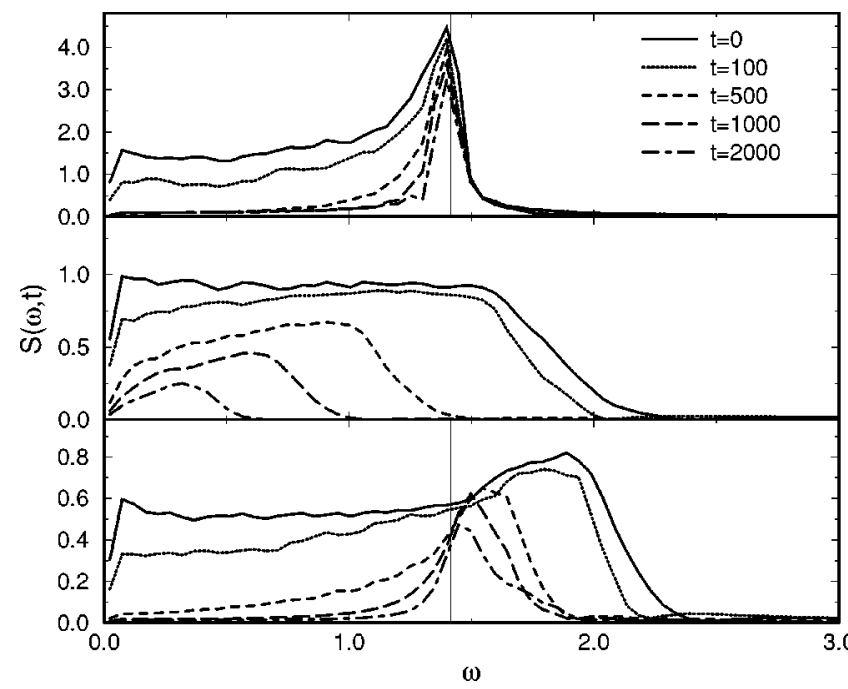

FIG. 2. Time evolution of spectra for various relaxing 1D arrays of 50 sites relaxing from a thermalized initial condition. In all cases the initial temperature is $T=0.5$, and $\gamma=0.1$. First panel: harmonic interactions $(k=0.5)$; second panel: purely anharmonic interactions $\left(k^{\prime}=0.5\right)$; third panel: mixed interactions $\left(k=k^{\prime}=0.5\right)$. The thin vertical lines indicate the harmonic frequency $\omega=\sqrt{4 k}=\sqrt{2}$. 


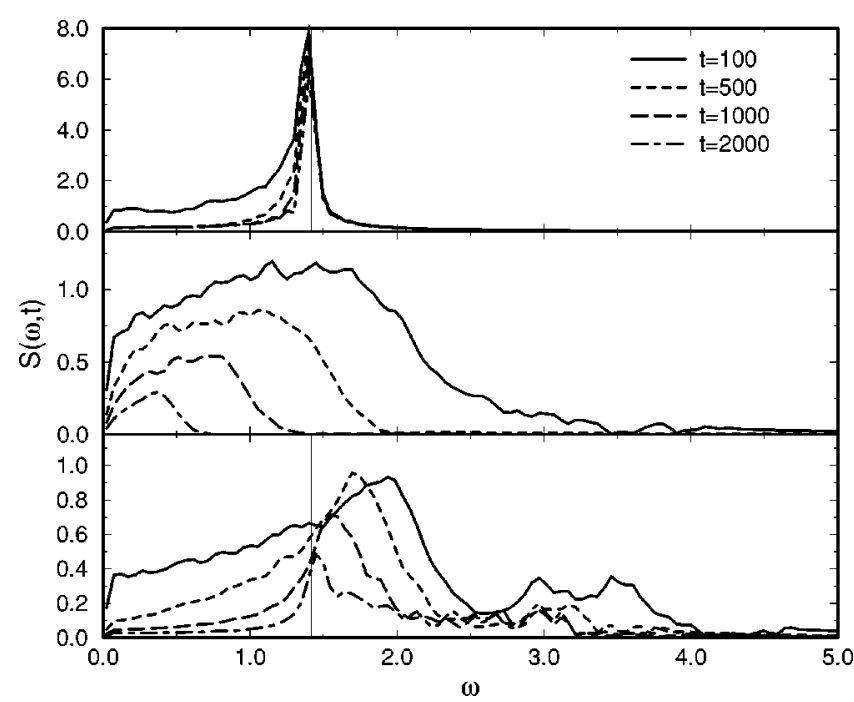

FIG. 3. Time evolution of spectra for various relaxing 1D arrays initially at $T=0.1$. At $t=0$ a high amplitude localized excitation is injected near the center of the chain. In all cases $\gamma=0.1$. First panel: harmonic interactions $(k=0.5)$; second panel: purely anharmonic interactions $\left(k^{\prime}=0.5\right)$; third panel: mixed interactions $\left(k=k^{\prime}=0.5\right)$. The thin vertical lines again indicate the harmonic frequency $\omega=\sqrt{4 k}=\sqrt{2}$.

harmonic chain at temperature $T$ and can be calculated analytically. ${ }^{19}$ We note that the only temperature dependence of this spectrum is an overall coefficient $T$. The progressive relaxation starting from the lower part of the spectrum and moving upward is clearly evident; by time $t=2000$ only the highest frequency phonons survive. The progression in twodimensions (2D) is very similar.

Although the above-mentioned analysis started from thermalized arrays, similar conclusions apply no matter the initial condition (including highly localized excitations), and no matter if and when additional excitations are injected in the array. ${ }^{14,15}$ Any distribution of energy consists of a superposition of phonons, and each phonon relaxes independently with its own characteristic decay rate. This is consistent with the spectral progression shown in the first panel of Fig. 3 . Here an odd parity localized excitation of amplitudes $-A / 2$, $A,-A / 2$ on three successive sites has been injected near the center of the thermalized chain at $t=0$. As before, the phonons decay progressively starting from the lower portion of the spectrum. The detailed $E(t)$ curve would of course be modified because the distribution of energy among the phonon modes is now different.

\section{NONLINEAR MODES}

As a second "extreme" case we consider a purely anharmonic array $(k=0)$. In the absence of harmonic forces the system supports no phonons, a condition that has been referred to as a sonic vacuum. ${ }^{20}$ This absence leads to a dynamic and relaxational behavior quite distinct from that observed in a mixed array (Sec. V), where phonons do constitute part of the spectrum. The precise nature of the full spectrum of excitations in this array is not known, but it certainly includes highly localized excitations. Indeed, breather solutions are exact in such chains when the power of the anharmonic potential (which here is 4) as well as $N$ go to infinity. $^{21}$

Consider first the relaxation of a purely anharmonic 1D array initially thermalized at temperature $T .{ }^{14}$ Although we do not study it here, we note that the temperature dependence of the relaxation process is more complex than in the harmonic array because now the frequencies of excitations depend very markedly on their energy. A characteristic of the purely anharmonic 1D array at any temperature is the essentially strictly exponential tail of the normalized energy $E(t)$. For example, the exponential decay of the purely anharmonic curves shown in Fig. 1 has been ascertained in detail in Ref. 14. This behavior implies that the purely anharmonic array approaches its new equilibrium much more rapidly than a harmonic system, and is indicative of a single predominant rate-limiting decay channel. Furthermore, the short time relaxation is more rapid with increasing temperature.

The spectral progression of the relaxation process is illustrated in the second panel of Fig. 2. Unlike the harmonic case, the equilibrium spectrum of the anharmonic chain broadens with increasing temperature because higher energy excitations involve higher frequencies. The higher frequency portions of the spectrum are observed to decay first, exactly opposite to the harmonic chain. We have found that the relaxation pathway is for the high frequency portions of the spectrum to degrade rapidly into lower frequency excitations; ${ }^{14}$ such a degradation pathway is possible here since individual frequencies are not associated with normal modes in the anharmonic system. In turn, these lower frequency excitations decay into the reservoir through the ends of the chain. The high frequency components of the spectrum are mainly associated with mobile localized modes that degrade into lower energy excitations (often also localized and mobile) as they move and collide with one another, and this degradation occurs relatively quickly. It is well known that higher frequency and/or higher amplitude localized modes can move at higher velocities. ${ }^{10-12,22}$ It is also known that while in motion such modes lose energy through collisions with other excitations. This picture is thus consistent with the more rapid decay of $E(t)$ with increasing temperature. The lower frequency excitations are in turn absorbed into the cold reservoir, but continue to be replenished through the degradation process. The decay of low frequency excitations into the reservoir dominate the exponential tail in the decay curves such as those seen in Fig. 1. We stress that this discussion only covers the predominant relaxation mechanisms. A concurrent direct but slow relaxation of high frequency excitations through the boundary sites may also take place. For example, when a highly mobile localized excitation reaches a boundary, it typically remains at the boundary for about one period of oscillation (which is short for a highly energetic excitation), during which it loses a small portion of its energy to the reservoir. The remaining excitation is reflected back into the chain, where it will continue to lose energy through other collisions and/or re-arrival at the boundaries.

The relaxation dynamics of the purely anharmonic array in 2D differs from the one-dimensional case in a number of 
ways. ${ }^{15}$ Mainly, the energy decay is considerably slower because localized excitations in 2D are not nearly as mobile as in $1 \mathrm{D}$; consequently, the energy loss caused by motion and by collisions is slowed down. Furthermore, the decay is no longer exponential. Indeed, whereas in one dimension the degradation process of higher frequency excitations into lower frequency ones is faster than the decay of low frequency excitations into the reservoir, in two dimensions this is no longer the case. This leads to spectral bottlenecks and competing time scales. We also find that increasing the array size leads to slower degradation of the high-frequency components and to more pronounced spectral bottlenecks in the midfrequency range. Still, with increasing initial temperature the total system energy decays more rapidly, which is consistent with our assertion that mobility (low as it may be) in the purely hard arrays increases with energy and hence with increasing initial temperature. We thus conclude that although breathers may be among the thermal excitations in purely nonlinear thermalized arrays, their rapid degradation makes it difficult to identify their precise dynamics.

In order to specifically focus on breather dynamics and the effects of other excitations on breathers, we inject a high amplitude localized excitation (one whose energy is much greater than those of the thermal excitations) at time $t=0$ and observe its evolution. Explicitly, we create an "odd parity" excitation with amplitudes $-A / 2, A,-A / 2$ on three successive sites away from the chain boundaries. Note that this is not an exact breather for this array, so some amplitude re-accommodation accompanied by some energy shedding necessarily takes place. The "bottom line" of this experiment is that the resulting breather is extremely robust in isolation, but extremely fragile when disturbed in any way. To see this, consider the case of such an excitation injected into a purely nonlinear array that has first been thermalized to temperature $T$. Although the details vary somewhat from one realization to another, it invariably happens very quickly that the other excitations in the medium set the breather in motion, and it loses energy mainly through its collisions with other excitations. A detailed observation of trajectories shows that after a short time the injected excitation begins to move in one direction or the other with equal probability, and continues moving for a period of random duration, during which it loses energy. The excitation stops moving for a random period of time, until it is again set in motion in either direction for another random period of time. While stationary, the excitation has even parity, but when it moves it alternates between even and odd parity. The collision partners in this sequence of events are principally the persistent lowfrequency excitations described earlier in the thermal relaxation process. ${ }^{14}$ Even while its amplitude and consequently its characteristic frequency are decreasing, the breather remains highly localized (over essentially three or four sites) throughout its lifetime. The spectral progression of the relaxation process is illustrated in the second panel of Fig. 3. In accordance with our description, it does not differ significantly from that of Fig. 2.

We have not measured the lifetime of the breather in this scenario, but note that it is much shorter than lifetimes to be reported later in mixed arrays, and varies quite a bit from one realization to another. It is, however, possible to follow the breather until it disappears, and to characterize its motion in terms of the mean square displacement $\left\langle x^{2}(t)\right\rangle$ of its center from its initial location. In 1D we find superdiffusive motion, $\left\langle x^{2}(t)\right\rangle \sim t^{1.5}$, over the entire lifetime of the excitation and for a variety of values of the coupling constant $k^{\prime}$, excitation amplitude, and temperature. ${ }^{14,15}$ Parameter variations seem only to affect the prefactor in this relation. It does not even matter when in the course of the relaxation process the localized excitation is introduced: its mean squared displacement grows with the same exponent, 1.5, until it is extinguished into the background. This corroborates the role of the persistent low-frequency excitations.

The situation is somewhat different in a purely anharmonic 2D array. Here we inject an excitation of amplitude $A$ at a central site and $-A / 4$ at each of the four nearestneighbor sites. It is more difficult in $2 \mathrm{D}$ to set a breather in motion since it is more difficult to bring about the symmetry breaking behavior that favors such motion (even parity breathers do not exist in 2D). This observation is consistent with our earlier comment concerning lower mobilities of breathers that may arise spontaneously in thermalized 2D arrays. Lower breather mobility of course does not preclude collisions with other mobile excitations that lead to energy loss. In any case, the breather survives for a longer time than it does in $1 \mathrm{D}$, and its mean square displacement is subdiffusive, $\left\langle r^{2}(t)\right\rangle \sim t^{0.89}$.

The scenario changes dramatically when the localized excitation is injected into a purely nonlinear chain that is initially at zero temperature. Breathers are almost exact stationary solutions in very long purely anharmonic chains regardless of the value of the damping parameter at the ends. In our simulations we inject an excitation of amplitude $A$ $=0.5$ near the chain center at time $t=0$ in a chain of $N$ $=31$ sites. For damping parameters $\gamma=0.01,1$, and 100 the energy remains essentially unchanged to all the controlled significant figures (12) for the duration of our simulation, $t$ $=3 \times 10^{6}$. We can also follow the motion of the sites surrounding the breather, and find that sites other than the three involved in the breather motion are almost stationary. Of course there must be an energy leakage out of the chain since equilibrium must eventually be reached, but for the chain length and simulation times in this particular experiment it was not discernible, so the decay is extremely slow. However, this longevity is, as before, fragile in that it is quickly destroyed by practically any perturbation. The source and nature of the perturbation does not much matter. A perturbation might arise, for example, when the slowly leaking energy reaches the chain boundaries and is partially reflected back toward the breather. This finite size effect should be observable if we shorten the chain sufficiently. For a chain of $N=17$ sites we still see no decay, but when we further shorten the chain to $N=15$ sites the breather survives almost undisturbed until a time of $\mathcal{O}\left(10^{6}\right)$ and then disintegrates rapidly, over a time scale of a few thousand time units. This is illustrated for one realization in Fig. 4. When $N=13$ the breather survives undisturbed for only about $7 \times 10^{3}$ time units and the decay occurs over a time period of about $10^{3}$.

Our conclusion is thus that breathers are exceptionally 


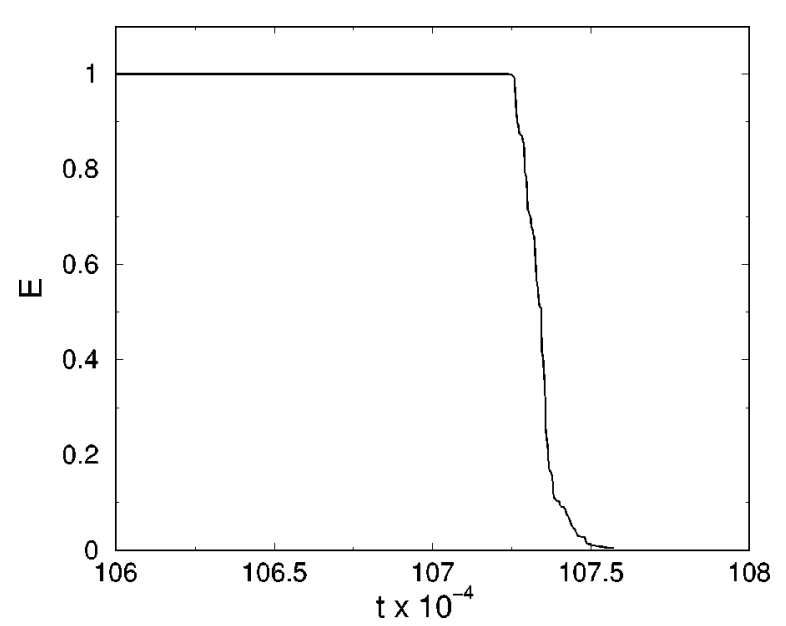

FIG. 4. Normalized energy for a purely anharmonic array $\left(k^{\prime}=0.5\right)$ of 15 sites in which a breather of amplitude $A=0.5$ has been injected into a zero temperature chain at $t=0$. The damping parameter $\gamma=1$.

stable in purely anharmonic arrays relaxing into a zero temperature heat bath through the array boundaries as long as there is no perturbation of any kind near the breather. Any perturbation, including even the smallest thermal perturbations or perturbations that reach the breather from the system boundaries, causes a rapid degradation of the breather.

\section{MIXED ARRAYS}

Mixed arrays, that is, arrays with both harmonic and anharmonic interactions, are the most versatile because they support both linear and nonlinear modes. The interplay of the two introduces new effects in the breather relaxation problem and a greater variability in breather dynamics. Also, the mixed array is representative of a larger variety of physical situations than the purely anharmonic. Furthermore, we will see that breathers may be exceptionally stable in relaxing mixed arrays. Therefore, a study of the full time evolution of the system during the relaxation process requires longer time histories than in the previous cases. Our discussion in this section deals only with 1D arrays because the short time behavior in $2 \mathrm{D}$ is very similar to that of the $1 \mathrm{D}$ systems, and because we have not carried out a long time study for the $2 \mathrm{D}$ system.

Again we begin with the relaxation of an array initially thermalized at temperature $T .{ }^{14}$ At low temperatures ( $T$ smaller than the phonon bandwidth associated with the linear portion of the potential) the relaxation is essentially identical to that of a harmonic chain. Interesting behavior clearly requires higher temperatures so that nonlinear excitations well above the phonon band can be part of the thermal mix. In Figs. 1 and 2 we observe the early time behavior of the energy relaxation and the spectral progression of the relaxation. The energy is at first seen to decay more rapidly than in the harmonic array. This is a consequence of the presence of both low-frequency phonons and high-frequency excitations in the system. Energy relaxation and decay thus involves both of the mechanisms discussed earlier, namely, that which characterizes the relaxation of the harmonic chain and, concurrently, that which characterizes the purely anhar-

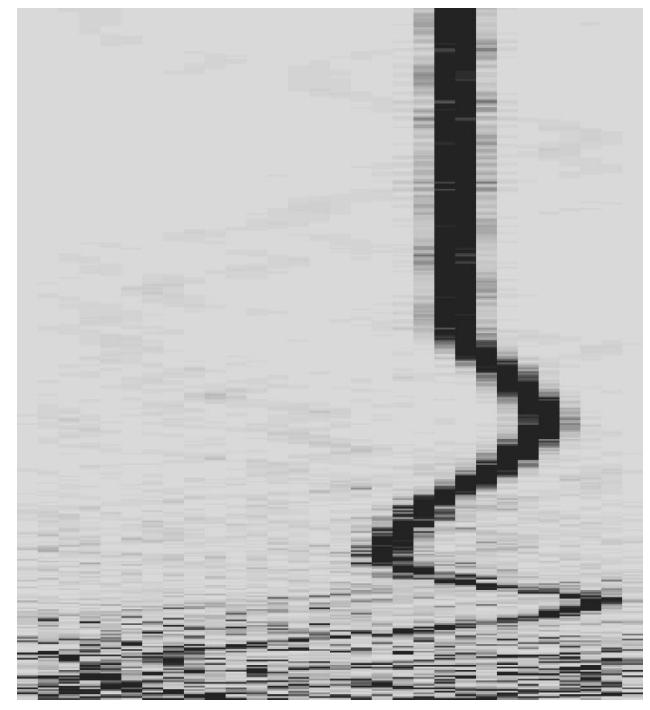

FIG. 5. Energy landscape of a 30-site mixed array initially thermalized at $T=0.5$. Other parameters: $k=k^{\prime}=0.5, \gamma=0.1$. Time advances along the $y$ axis until $t=1000$. A gray scale is used to represent the local energy, with darker shading corresponding to more energetic regions.

monic chain. Again, because initially the high-frequency modes move more rapidly at higher temperatures, the early time decay is faster with increasing initial temperatures. That both low and high frequency modes relax rapidly is clearly seen in the third panel of Fig. 2, which quickly loses both low (as in the first panel) and high (as in the second panel) frequency portions of the spectrum. In the energy decay curve there is then a crossing after which the mixed chain relaxes much more slowly than the harmonic and the purely anharmonic. This occurs when the low frequency modes (phonons) have essentially all decayed, and only certain high frequency spectral components remain, as clearly seen in the spectrum.

The excitations that remain after this initial shedding include, with some probability that depends on temperature (but not with certainty), spontaneously created quasistationary breathers that decay extremely slowly. It is a noteworthy reminder that short wavelength zone-boundary phonons that relax much more slowly than long wavelength phonons actually contribute to the spontaneous formation of breathers ("modulational instability"). ${ }^{23}$ The decay of the breather is slower with increasing temperature because the spontaneously created breathers are more energetic. A typical realization in which a breather appears spontaneously is shown in Fig. 5. In stark contrast to the purely anharmonic array, there is no sonic vacuum in the mixed array. The harmonic contribution to the interaction, which provides the phonon excitations, allows the relaxation process to "sweep" the system clean of the excitations that most readily perturb the breather, and thus makes it possible for the breather to persist in the relaxing environment.

Next we examine in detail the continued evolution of a spontaneously created breather in our relaxing environment, ${ }^{16}$ going to much longer times in our simulations than those reported so far in order to ascertain the relaxation behavior in more detail. We distinguish the normal- 

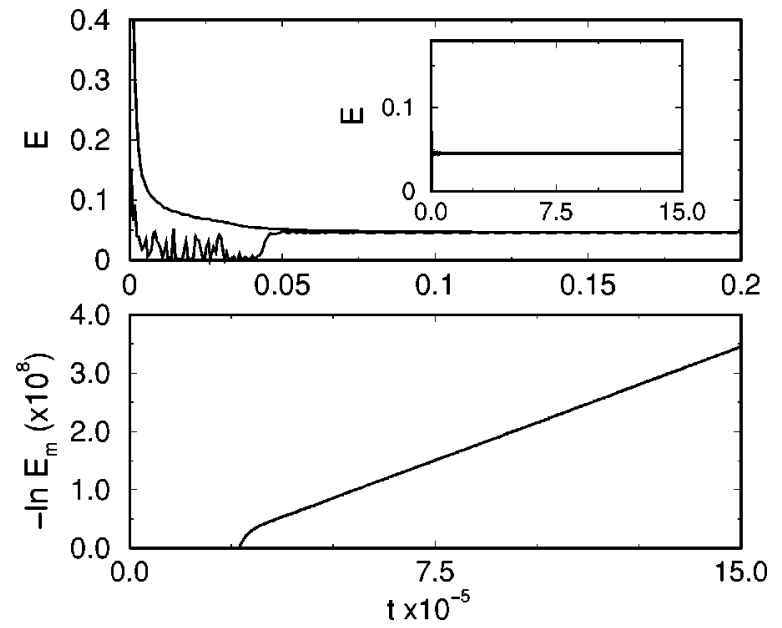

FIG. 6. Upper panel: the smooth curve is the normalized energy as a function of time for a chain of 30 sites initially thermalized at $T=0.5$ and connected through its ends to a zero-temperature heat bath $(\gamma=0.1)$. The initially jagged curve is the normalized energy on sites $13,14,15$, and 16 . The inset shows the temporal evolution of the energy on these four sites over a longer time scale. Lower panel: $\left[-\ln E_{m}(t)\right]$ vs $t$ for the same chain.

ized energy $E(t)$ [cf. Eq. (7)] from the modified normalized energy $E_{m}(t)$

$$
E(t)=\frac{\varepsilon(t)}{\varepsilon(0)}, \quad E_{m}(t)=\frac{\varepsilon(t)}{\varepsilon\left(\tau_{m}\right)} .
$$

The denominator in the first contains the initial energies, and in the second the energies after the discarded low energy excitations (including all phonons) have dissipated, but before the remaining breather has decayed appreciably. In our simulations we take $\tau_{m}=40000$. Figure 6 shows the evolution of the normalized energy in a chain of 30 sites initially thermalized at temperature $T=0.5$, as well as the energy in only the four sites $i=13,14,15,16$. After a relatively short time (5000 time units in this particular realization) almost all of the energy settles in these sites and remains there. The excitation around the four sites turns out to be an "even parity" breather, with maximum displacements $A$ and $-A$ alternating on sites 14 and 15 , smaller but not negligible amplitudes at sites 13 and 16, and essentially no motion at the other sites. The breather is coincidentally at the center of the chain, but may appear anywhere in different realizations (cf. Fig. 5), particularly in longer chains. The frequency of the breather, initially $\omega=1.633$, decreases very little for the duration of the simulation. We also present the modified normalized energy, whose decay is clearly exponential over the times shown, with an enormously long time constant, $\tau$ $=3.5 \times 10^{13}$. Thus this breather, even in our relatively short chain, is essentially stationary.

In order to ascertain our breather stability scenario and the role of phonons in the stability, we explicitly inject a breather into a chain that is in thermal equilibrium at a very low temperature (low in the sense that the spontaneous formation of breathers is highly unlikely). The chain is then allowed to relax into a zero temperature heat bath (cf. third panel in Fig. 3). As expected, we find that the thermal background invariably sets the breather in motion, and causes the breather to collide with other excitations and with the chain boundaries. The resulting decay of the breather is then in general much faster than in the scenario where the breather is created spontaneously during the relaxation process (and certainly much faster than that of a breather of the same amplitude injected into a zero temperature chain, cf. the following). We find this behavior even when the temperature is extremely low. For example, a breather of initial amplitude $A=0.5$ injected into a chain thermalized at $T=10^{-6}$ has a decay time of $\tau=1.3 \times 10^{6}$ (the value varies from one realization to another, but not by much). With an initial amplitude of $A=0.55$ we find $\tau=2.1 \times 10^{6}$. Note that it does not much matter whether the injected breather is of even or odd parity (here we have injected an odd one) ${ }^{24}$ As we will see in the following, although the initial temperature is extremely low, these decay times are orders of magnitude shorter than those of a breather injected in a zero temperature chain, the crucial difference being the presence of low wavelength phonons in the former but not in the latter. To support this description we have also observed a breather in a chain connected to a heat bath that is maintained at an extremely low but nonzero temperature. The breather in this case is always fragile, continuing to move and lose energy rapidly until it degrades completely.

We now move on to our second scenario, namely, we inject the excitation into a chain at zero temperature. ${ }^{16} \mathrm{Al}-$ though we have not done so in detail in the cases presented so far, here we have carried out a more detailed analysis of dependencies of the breather dynamics and relaxation on a number of parameters. The results are revealing and, in some cases, somewhat unexpected. In our first "experiment" we create an odd-parity excitation of amplitude $A$ exactly in the middle of a chain of $N=31$ sites, and set the end-site dissipation parameter $\gamma=1$. The breather discards some energy that travels toward the chain ends and dissipates quickly, in the time $\tau_{m}$ [cf. Eq. (8)], across the ends of the chain. The remaining energy stays localized in the middle of the chain, most of it $(98 \%$ for $A=0.5)$ on the three initially excited sites, and decays exponentially with an extremely long decay time $\tau$.

In Fig. 7 we show typical results for $E(t)$ for three excitation amplitudes over more than six decades of time. The modified normalized energy follows essentially the same behavior. If the decay of the long-lived excitation is exponential, we expect [ $-\ln E(t)]$ and $\left[-\ln E_{m}(t)\right]$ vs $t$ to be straight lines over appropriately long time intervals. In Fig. 8 we clearly see this behavior, which extends over the entire simulation time interval for the higher amplitude excitation. The slope for the $A=0.5$ curve leads to a decay time of $\tau=2.8$ $\times 10^{9}$, a specific number reported here principally to stress its enormous magnitude compared to phonon relaxation times. The change in slope of the curve associated with the lower amplitude breather captures the slow change in the decay rate as the breather frequency edges toward the phonon band. Here we also see clearly that the more energetic breather relaxes more slowly.

A breather of a given amplitude has a characteristic predominant frequency. In Fig. 9 we show this frequency in relation to the phonon band edge as a function of time for various cases. For 31-site chains, the frequency of the 


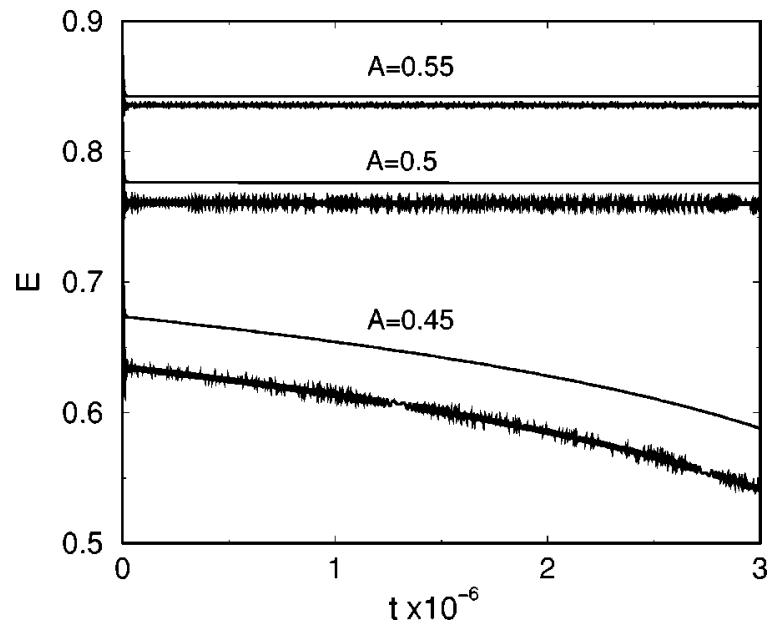

FIG. 7. Decay of the normalized energy $E(t)$ for three different values of the initial amplitude $A$ for chains of $N=31$ sites connected at the ends to a zero-temperature bath. The odd-parity breather is injected at the center of the chain. The dissipation parameter $\gamma=1$. The thin lines represent the total energy remaining in the chain, and the bold lines the portion of the remaining energy that is localized on the three initially excited sites.

breather of initial amplitude $A=0.5$ decreases very little over the entire simulation, while that of initial amplitude $A$ $=0.45$ decreases more markedly. Consistent with the fact that the breather does not disappear entirely in the time range shown, its frequency never reaches the phonon band edge. If the initial amplitude of the excitation is sufficiently low, or the simulation time sufficiently long, or the chain sufficiently short, the breather is seen to disappear. This last case is illustrated in the figure for a breather of initial amplitude $A$ $=0.5$ in a 21 -site chain. The breather disintegrates entirely when its frequency reaches the phonon band edge. The inset shows $L$, the ratio of the energy of the five sites centered on the breather to the total energy. $L$ is of order unity when most of the energy is localized on a small number of sites. Note that the lifetime of this breather, which is of $O\left(5 \times 10^{5}\right)$, is still much longer than the longest phonon lifetime, which is of $O\left(10^{4}\right)$.

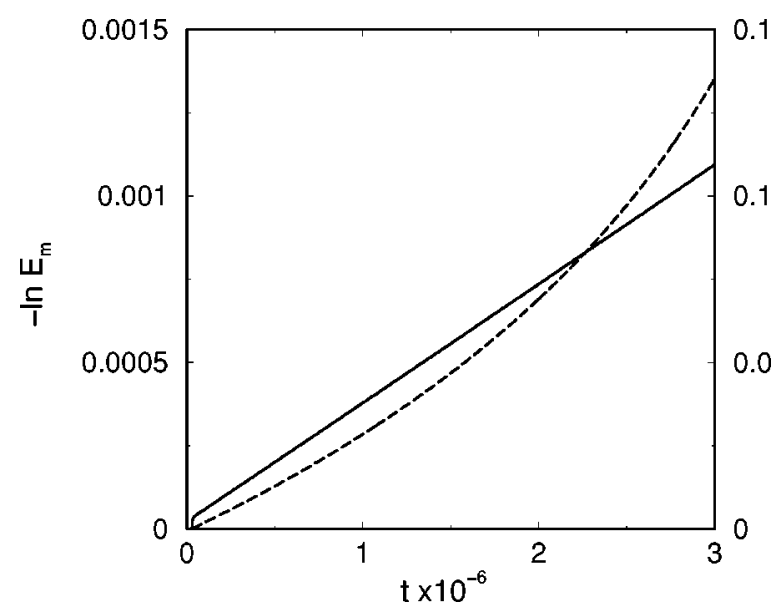

FIG. 8. [ $-\ln E_{m}(t)$ ] vs $t$ for two initial amplitudes, $A=0.5$ (solid curve, left scale) and $A=0.45$ (dashed curve, right scale), for a chain of 31 sites with an odd-parity breather at the center and $\gamma=1$.

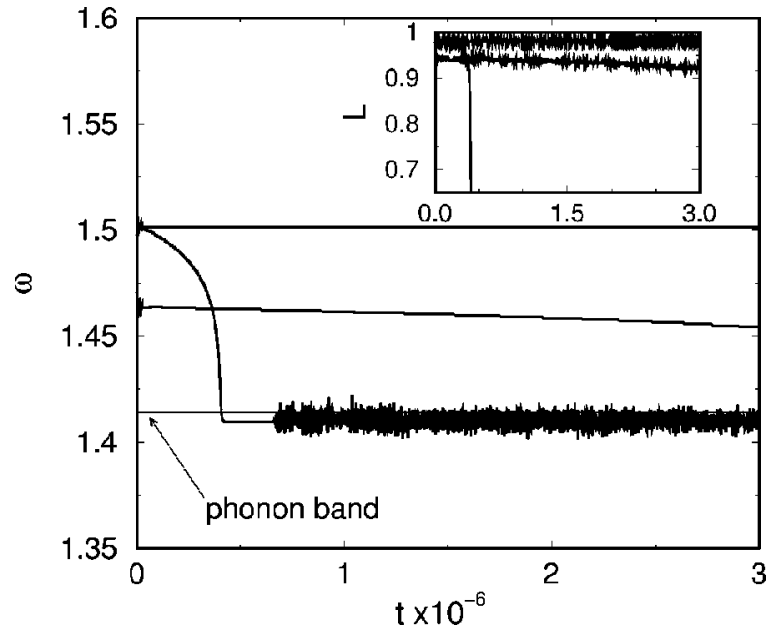

FIG. 9. Breather frequency as a function of time. Curve that persists at the highest frequency $(\omega \sim 1.5): A=0.5, N=31$. Curve that begins at $\omega$ $\sim 1.465$ and decreases gently: $A=0.45, N=31$. Curve that turns sharply downward: $A=0.5, N=21$. Inset: associated localization parameters in the same order.

The above-mentioned results are typical of one particular set of conditions: a breather created exactly in the middle of a chain of $N$ sites whose ends are connected to a zerotemperature bath with dissipation parameter $\gamma=1$. It is interesting to explore the consequences of changing some of these conditions. We find that the dependence of the chain energy relaxation times on the initial amplitude of the breather is monotonic and decreases sharply with decreasing breather amplitude. Over a simulation time of $3 \times 10^{6}$ we find that a breather of initial amplitude $A=0.6$ decays exponentially with a time constant $\tau=2.8 \times 10^{14}$. For amplitude $A=0.5$ we find $\tau=2.8 \times 10^{9}$, and for $A=0.45$ the decay is no longer strictly exponential, decreasing slightly from 3.0 $\times 10^{7}$ to $1.5 \times 10^{7}$ over the course of the simulation.

The evolution of the breather depends in an interesting way on its initial location and on the damping parameter $\gamma$ when the latter is either very small or very large. Figure 10 shows the early evolution (up to $t=2000$ ) of an initially slightly off-center breather (site 15 of a 31-site chain), for three values of the damping parameter. The middle panel is for $\gamma=1$, the damping we have considered so far. The behavior of the excitation in this panel starts out as we have described it, that is, it sheds some energy (medium-gray scale) that dissipates quickly. Although a small fraction of the energy that has been shed returns toward the breather, it is neither sufficient nor of the long wavelength variety to set it in motion; most of the shed energy simply dissipates into the zero-temperature bath. The evolution of the breather proceeds much like that of a breather initially centered in the middle of the chain (site 16), with only a small modification of its decay time. This behavior is fairly robust for values of $\gamma$ within an order of magnitude on either side of $\gamma=1$ and for breathers that are excited not too near the chain ends.

The situation is rather different if $\gamma$ is either very small (first panel) or very large (third panel). The qualitative similarity between these two extreme cases is apparent, and consistent with our discussion of high and low damping simi- 

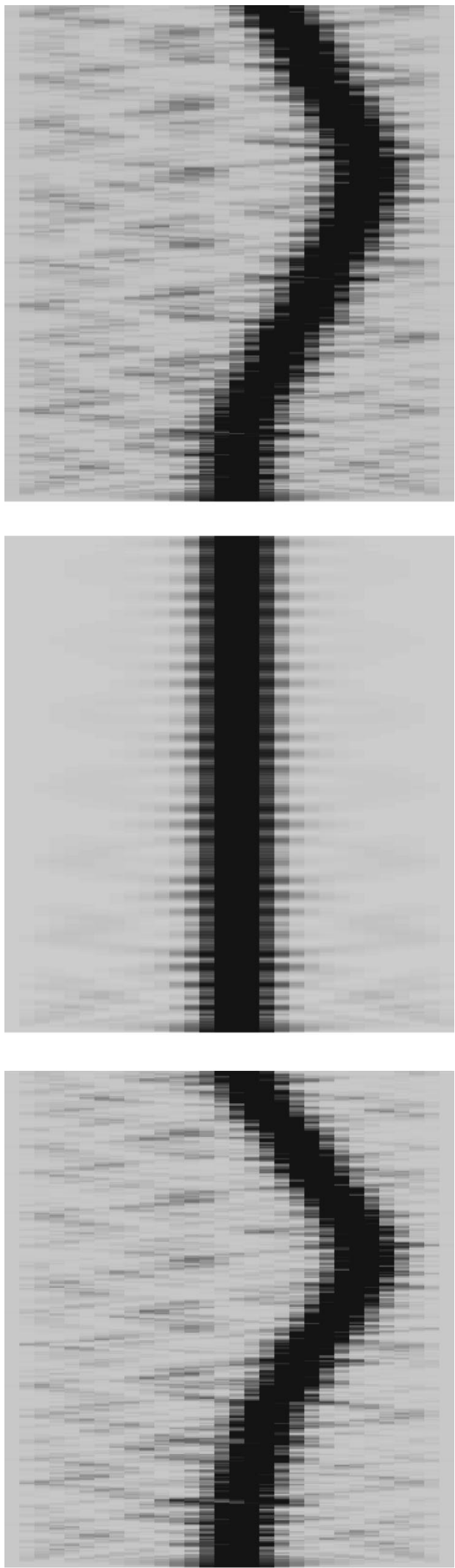

FIG. 10. Energy landscapes of 31-site arrays. The injected odd-parity breather of amplitude $A=0.5$ is initially centered at site 15 . Time advances along the $y$ axis until $t=2000$. A gray scale is used to represent the local energy, with darker shading corresponding to more energetic regions. First panel: $\gamma=0.01$. Second panel: $\gamma=1$. Third panel: $\gamma=100$. larities in a purely harmonic chain; the chain ends no longer effectively dissipate the energy that has been shed by the breather, and so it returns to perturb the breather and set it in motion. In turn, this causes the breather to decay more rapidly into more rapidly dissipated lower-energy excitations. ${ }^{16}$ In the very low damping case, energy that arrives at the chain ends cannot go anywhere except back, much like a whip. In the very high $\gamma$ case the end sites are so damped that they can absorb very little energy from the rest of the chain, much like a wall. Indeed, the very low $\gamma$ case is very similar to a dissipationless chain with periodic boundary conditions, while the very high $\gamma$ case is akin to a dissipationless chain with fixed boundary conditions. The former has been studied in detail in Refs. 10 and 11 and the latter in Ref. 12. These papers study the formation and evolution of breathers in the process of relaxation to thermal equilibrium (equipartition) in dissipationless chains with nonequilibrium initial conditions, and particularly focus on the interesting emergence of chaotic breathers in this relaxation process. The point of interest here is that the dynamics toward equipartition in these systems is rather insensitive to the boundary conditions, an observation consistent with the similarity of our very low and very high $\gamma$ results.

We have followed these particular histories over our usual time span of 3 million time units and find the decay times $\tau=\mathcal{O}\left(10^{9}\right)$ for $\gamma=1, \mathcal{O}\left(10^{6}\right)$ for $\gamma=0.01$, and $\mathcal{O}(3$ $\times 10^{6}$ ) for $\gamma=100$. The specific values change depending on the initial location of the breather and the values of the other parameters of the system, but the trend is clear.

An odd parity breather initially centered exactly in the middle of the chain constitutes a singular case when damping is very low or very high, with relative decay rates opposite to those reported earlier. While the $\gamma \sim 1$ results are not much affected by the initial location of the excitation (provided it is far from the chain ends), in this peculiar case the extreme- $\gamma$ cases lead to slower decay than for $\gamma \sim 1$. In this uniquely symmetric case, the breather is perturbed from both sides by identical energy pulses that return from the ends of the chain. In the absence of symmetry breaking, the breather is therefore not set in motion, and instead simply re-absorbs this energy (and re-emits and re-absorbs energy in increasingly smaller amounts). Since the energy that returns from the chain ends is greater in the extreme $\gamma$ cases than it is for intermediate $\gamma$, the chain energy remains higher, and the decay is thus slower.

Breather decay times are strongly dependent on chain length: the decay times increase markedly, as does the total lifetime of the breather, with increasing $N$ because finite size effects and disturbances scattered back from chain boundaries are reduced. This is already apparent when one compares the $N=31$ and $N=21$ results in Fig. 9. Whereas a breather of initial amplitude $A=0.5$ created at the center of a 31 -site chain has barely decayed over 3 million time units, a breather of the same initial amplitude in a 21-site chain has disintegrated completely well before that. With $A=0.5$ and $\gamma=1$ for the centered breather we find $\tau=2.8 \times 10^{9}$ for $N$ $=31$ (as reported earlier), $\tau=3.2 \times 10^{12}$ for $N=41$, and $\tau$ $=3.6 \times 10^{15}$ for $N=51$.

Exponential decay points to a single rate-limiting decay 
channel for the energy. This channel is the shedding of energy in the form of phonons and/or lower energy localized excitations by the breather. The degradation of lower-energy localized excitations, and the dissipation of energy into the zero-temperature bath, are much faster processes. However, the relaxation rate associated with the shedding process is strongly dependent on chain length, breather location, and other system parameters.

To tie together all the scenarios that we have presented in support of our picture of breather dynamics in mixed arrays, we add one more "experiment:" we follow the dynamics of a breather injected into a relaxing chain after the long wavelength phonons have decayed, but before the thermal relaxation process is complete. If our picture is correct, the breather lifetime should be much longer than that of one injected at time $t=0$ (albeit perhaps shorter than that of the same breather injected in a zero temperature chain). We do indeed find that the breather stability improves dramatically. For example, for a zero-temperature injected breather of initial amplitude $A=0.6$ in a chain of 31 sites we reported earlier that over 3 million time units the relaxation time of the breather is $\tau=2.8 \times 10^{14}$. In a chain initially thermalized at $T=10^{-5}$ and then allowed to relax, if we wait until $t$ $=15000$ before injecting the same breather we find a somewhat shortened but still very long decay time of $\tau=5.9$ $\times 10^{13}$, in any case much longer than it would be if injected at $t=0$.

We end this section with a caveat: all the exponential and quasiexponential slow decays reported for the various scenarios are for single realizations. In thermalized scenarios where breathers are created spontaneously (but not necessarily in every realization), an ensemble average could lead to a time dependence of the array energy that may be complicated by the occurrence of a broad range of relaxation times. In the other scenarios, e.g., where breathers are injected "manually," a range of relaxation times might also occur in an ensemble if the location of the breather varies from one realization to another.

\section{CONCLUSIONS}

We have studied the dynamics and relaxation of breathers in Fermi-Pasta-Ulam arrays whose boundaries are connected through damping terms to a zero temperature heat bath. We find that breather dynamics and relaxation in these nonlinear arrays with quartic inter-particle interactions proceed along energetic pathways that are highly sensitive to the presence or absence of quadratic contributions to the interactions.

To understand the role of quadratic interactions we have recalled that phonons in these arrays relax independently of one another (provided the damping at the boundaries is not too strong), that the phonon relaxation time is wave vector dependent, and that phonons therefore relax sequentially, starting with the longest wavelengths for the free-end boundary conditions used in our work. We have also pointed out that breathers are fragile against collisions with long wavelength phonons and also with other localized nonlinear excitations. Breathers are therefore quite robust in the absence of long wavelength phonons and of other nonlinear excitations, but are rapidly degraded in the presence of either. To arrive at these conclusions, and to investigate them more quantitatively (at least numerically), we have performed a number of numerical experiments involving the spontaneous and the manual creation of breathers in arrays initially at finite temperatures and at zero temperature.

Breather decay brought about by collisions with long wavelength phonons and with other nonlinear excitations, and by the associated breather motion, is rapid, even more rapid than typical relaxation times of high frequency phonons. The actual process of breather disintegration caused by collisions and associated motion is one whereby the breather breaks up rapidly into lower energy excitations. These mechanisms of breather decay cause their lifetimes to be short in systems that contain such excitations. Examples include thermalized purely anharmonic arrays that have no efficient way to eliminate their thermal excitations. Even at zero temperature, a manually injected localized mode in a purely anharmonic array will (perhaps after a prolonged period of stability) eventually succumb rather suddenly and rapidly to the very perturbations produced during the relaxation process as the localized mode re-accommodates itself and/or the energy it sheds is reflected back by the system boundaries (finite size effects). Breather decay is also rapid if a breather is manually injected in a thermalized mixed array, mainly due to the effects of long wavelength phonons. Since these phonons are highly destructive of breathers, breather degradation is observed even when the temperatures involved are extremely low.

On the other hand, breathers that are isolated from the effects of long wavelength phonons and of other nonlinear excitations persist for extremely long times. Examples are spontaneous breathers that arise during thermal relaxation of a mixed array. Long wavelength phonons as well as other nonlinear excitations that themselves decay into phonons are the first to relax, and spontaneous breathers make their appearance when the system has already been swept clean of these particular excitations. Short wavelength phonons do not destroy breathers; on the contrary, they tend to be absorbed by them and thus to contribute to their stability. The crucial importance of phonons and their ability to relax into the cold temperature heat bath (especially the long wavelength phonons) is thus evident: spontaneously created breathers in mixed arrays can persist because of the phonon dynamics, whereas the absence of phonons (sonic vacuum) and the consequent difficulty of purely anharmonic arrays to eliminate "offending" excitations leads to the inability of spontaneously created breathers to persist. For the same reasons, manually injected breathers in mixed arrays can persist for a very long time if inserted in a zero temperature array, or in an array in which long wavelength phonons and other nonlinear excitations have already relaxed, but breathers will not survive if injected in a thermalized mixed chain, or even in a chain that is allowed to cool down to a very low but nonzero temperature, no matter how low the temperature.

Having established conditions that favor breather longevity (mixed anharmonic chains at zero temperature or partially relaxed to zero temperature), we have noted that even 
these breathers must eventually relax through some intrinsic energy shedding process since the chain must eventually equilibrate to zero temperature. This intrinsic process is very slow and essentially exponential over very long periods of time, although some deviation from strict exponentiality is eventually observed because the relaxation time is amplitude dependent. Thus, as the breather slowly loses energy/ amplitude, this "single" relaxation time necessarily decreases. This quasiexponential decay continues until the breather frequency (which decreases with decreasing amplitude) approaches the phonon band, at which point the breather quickly breaks up into phonons that decay rapidly. The very slow quasi-exponential decay is indicative of essentially a single leakage channel. We have observed that the slow decay rates are dependent on system parameters, on breather location, and on breather amplitude. Therefore, whereas single observations of energy relaxation of systems supporting one long-lived breather will lead to essentially exponential decay over many decades of time, ensemble averages might show more complex behavior. This is a specially strong caveat in experiments involving spontaneous breather creation and the associated possibility of realizations in which no breathers occur at all.

\section{ACKNOWLEDGMENTS}

This work was supported by the Engineering Research Program of the Office of Basic Energy Sciences at the U. S. Department of Energy under Grant No. DE-FG0386ER13606. Support was also provided by a grant from the University of California Institute for México and the United States (UC MEXUS) and the Consejo Nacional de Ciencia y Tecnología de México (CoNaCyT).
${ }^{1}$ S. Flach and C. R. Willis, Phys. Rep. 295, 181 (1998); Physica D 119, 1 (1999)

${ }^{2}$ S. Aubry, Physica D 103, 201 (1997).

${ }^{3}$ M. Peyrard and J. Farago, Physica A 288, 199 (2000).

${ }^{4}$ G. Kopidakis and S. Aubry, Physica B 296, 237 (2001).

${ }^{5}$ T. Rössler and J. B. Page, Phys. Rev. B 62, 11460 (2000).

${ }^{6}$ S. F. Mingaleev, Y. S. Kivshar, and R. A. Sammut, Phys. Rev. E 62, 5777 (2000)

${ }^{7}$ G. Kopidakis, S. Aubry, and G. P. Tsironis, Phys. Rev. Lett. 87, 165501 (2001)

${ }^{8}$ N. J. Zabusky and M. D. Kruskal, Phys. Rev. Lett. 15, 240 (1965); F. Zhang, D. J. Isbister, and D. J. Evans, Phys. Rev. E 61, 3541 (2000).

${ }^{9}$ V. M. Burlakov, S. A. Kiselev, and V. N. Pyrkov, Phys. Rev. B 42, 4921 (1990); R. Dusi, G. Viliani and M. Wagner, Philos. Mag. B 71, 597 (1995); Phys. Rev. B 54, 9809 (1996); Y. A. Kosevich, ibid. 47, 3138 (1993).

${ }^{10}$ Y. A. Kosevich and S. Lepri, Phys. Rev. B 61, 299 (2000).

${ }^{11}$ T. Cretegny, T. Dauxois, S. Ruffo, and A. Torcini, Physica D 121, 109 (1998).

${ }^{12}$ V. V. Mirnov, A. J. Lichtenberg, and H. Guclu, Physica D 157, 251 (2001).

${ }^{13}$ A. J. Sievers and S. Takeno, Phys. Rev. Lett. 61, 970 (1988); J. B. Page, Phys. Rev. B 41, 7835 (1990); K. W. Sandusky, J. B. Page, and K. E. Schmidt, ibid. 46, 6161 (1992); T. Dauxois and M. Peyrard, Phys. Rev. Lett. 70, 3935 (1993); S. Aubry, Physica D 71, 196 (1994); R. S. MacKay and S. Aubry, Nonlinearity 7, 1623 (1994); D. Cai, A. R. Bishop, and N. Gronbech-Jensen, Phys. Rev. E 52, 5784 (1995); S. Takeno and M. Peyrard, Physica D 92, 140 (1996).

${ }^{14}$ R. Reigada, A. Sarmiento, and K. Lindenberg, Phys. Rev. E 64, 066608 (2001)

${ }^{15}$ R. Reigada, A. Sarmiento, and K. Lindenberg, Physica A 305, 467 (2002).

${ }^{16}$ R. Reigada, A. Sarmiento, and K. Lindenberg, Phys. Rev. E 66, 046607 (2002).

${ }^{17}$ F. Piazza, S. Lepri, and R. Livi, J. Phys. A 34, 9803 (2001).

${ }^{18}$ Our thermalization methods, which involve adding Langevin forces to all the equations of motion, are detailed in our earlier work, Refs. 14-16.

${ }^{19}$ R. Reigada, A. Sarmiento, and K. Lindenberg, Phys. Rev. E 63, 066113 (2001)

${ }^{20}$ S. L. Gavrilyuk and V. F. Nesterenko, J. Appl. Mech. Tech. Phys. 34, 784 (1993); V. F. Nesterenko, J. Phys. IV 4, C8/729 (1994).

${ }^{21}$ S. Flach, Phys. Rev. E 51, 1503 (1995); J. L. Marin and S. Aubry, Nonlinearity 9, 1501 (1996).

${ }^{22}$ R. Bourbonnais and R. Maynard, Int. J. Mod. Phys. C 1, 233 (1990).

${ }^{23}$ I. Daumont, T. Dauxois, and M. Peyrard, Nonlinearity 10, 617 (1997).

${ }^{24}$ J. B. Page, Phys. Rev. B 41, 7835 (1990); K. W. Sandusky, J. B. Page, and K. E. Schmidt, ibid. 46, 6161 (1992); Y. S. Kivshar et al., Phys. Rev. E 58, 5057 (1998) 